\title{
Addressing the challenge of integrating research advances into patient care
}

\author{
Robert W Schrier
}

For the busy clinician, keeping abreast of the myriad advances in cellular and molecular medicine is a formidable challenge. Nature Clinical Practice Nephrology addresses this challenge. This new journal provides the practicing physician with a comprehensive overview of the most up-to-date literature relevant to diagnosis and management of patients with kidney disease. All full members of the International Society of Nephrology will receive the journal as a valuable benefit of membership.

Staff of, and contributors to, Nature Clinical Practice Nephrology filter a vast amount of literature from a wide range of publications. Information with the greatest clinical implications for all subspecialties is then selected, interpreted and presented in a uniquely digestible format. A product of the renowned publishers of Nature that harnesses the expertise of an eminent international Advisory Board, Nature Clinical Practice Nephrology is an exciting educational vehicle that will ease the workload of nephrologists and affiliated healthcare professionals.

Nature Clinical Practice Nephrology's succinct and uniquely tailored articles are written by experts and professionally edited for maximum impact. Each monthly issue is prefaced by an Editorial, written either by myself, a member of the Advisory Board or their nominated representative. Viewpoints present the opinions of high-profile specialists on important and often controversial issues that confront the nephrology community. Snapshots of several recently published original or evidence-based articles, compiled by the in-house editorial team, comprise the Research Highlights section. The thorough literature searching undertaken by our staff ensures coverage of clinically relevant work from general medical journals and leading nephrology publications, as well as high quality research from journals that publish relevant content on an infrequent basis.

Practice Points are two-page articles consisting of a structured summary of an

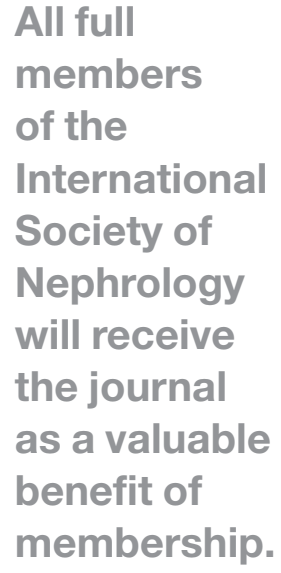

RW Schrier is Editorin-Chief of Nature Clinical Practice Nephrology, and former Chairman of the Department of Medicine at the University of Colorado School of Medicine.

\section{Competing interests}

The author declared he has no competing interests.

www.nature.com/clinicalpractice doi:10.1038/ncpneph0015 original research paper and a commentary from an authority in the field. The commentary places the research in clinical context and is accompanied by a single-sentence recommendation of how the new findings should affect nephrologists' care of their patients. In addition to General Reviews, several other Review types-Mechanisms of Disease, Drug Insights, Technology Insightsprovide timely and authoritative overviews of recent developments. Therapy Insights facilitate an interdisciplinary outlook. Reviews of this type discuss conditions managed by physicians in other specialties that are relevant to nephrologists; for example, comorbidities such as cardiorenal and hepatorenal syndromes, described by a cardiologist and a hepatologist. Rounding out each monthly issue is a Case Study that comprehensively describes the diagnosis and management of patients who present with an unusual history, symptoms or physical findings. More straightforward cases that were managed using new technologies and techniques are also presented.

All content of Nature Clinical Practice Nephrology is rigorously peer reviewed (with the exception of Research Highlights and Editorials), and all authors are requested to declare professional or financial interests that may be perceived as a conflict of interest with respect to the integrity of their contribution. Further value, in the form of an opportunity to earn Category $1 \mathrm{CME}$ credits, comes via our partnership with an ACCME-accredited provider.

I am delighted to be the Editor-in-Chief of this exciting new venture. It is a pleasure to work with the Nature Publishing Group, which is soon to become publisher of Kidney International. I believe Nature Clinical Practice Nephrology will be a great asset to practicing nephrologists in their effort to maintain and improve their knowledge, thereby enhancing care of their patients. 